\title{
Raman spectroscopy and instrumentation for monitoring soil carbon systems
}

\author{
D. L. Stokes, S. Wullschleger, M. Martin and T. Vo-Dinh* \\ Oak Ridge National Laboratory; P.O. Box 2008; Oak Ridge, TN 37831-6101
}

\begin{abstract}
This work describes developments in the application of Raman scattering and surface-enhanced Raman scattering (SERS) towards the assessment/characterization of carbon in soil. In the past, the nonspecific total carbon mass content of soil samples has generally been determined through mass loss techniques and elemental analysis. However, because of the concern over $\mathrm{CO}_{2}$ buildup in the atmosphere and its possible role in the "Greenhouse Effect," there is a need for better-defined models of global cycling of carbon. As a means towards this end, there is a need to know more about the structure and functionality of organic materials in soil. Raman spectroscopy may therefore prove to be an exceptional tool in soil carbon analysis. Based on vibrational transitions of irradiated molecules, it provides structural information that is often suitable for sample identification. Furthermore, Raman scattering yields very fine spectral features which offer the potential for multicomponent sample analysis with minimal or no sample pretreatment. Although the intensity of Raman scattering is generally extremely low, the surface-enhanced Raman scattering (SERS) effect can greatly enhance Raman signals $\left(10^{6}-10^{8}\right.$ range) through the adsorption of compounds on specially roughened metal surfaces. In our laboratory, we have investigated copper, gold and silver as possible substrate metals in the fabrication of SERS substrates. These substrates have included metal-coated microparticles, metal island films, and redox-roughened metal foils. We have evaluated several laser excitation sources spanning the 515-785 nm range for both Raman and SERS analysis. For this particular study, we have selected fulvic and humic acids as models for establishing the feasibility of using Raman and SERS in soil carbon analysis. Our studies thus far have demonstrated that copper substrates perform best in the SERS detection of humic and fulvic acids, particularly when coupled to electrochemical processes that enhance adsorption of specific compounds. This effect not only yields a stronger signal, but can also impart selectivity in the analysis of complex samples such as soil.
\end{abstract}

Keywords: Raman, surface-enhanced Raman scattering (SERS), carbon sequestration, humic acid, fulvic acid, p-aminobenzoic acid, brilliant cresyl blue, copper substrate, silver substrate

\footnotetext{
* -author to whom correspondence should be sent
} 


\section{INTRODUCTION}

Raman spectroscopy may prove to be a valuable tool in a research area of ever-growing importance, characterization of soil carbon systems. This consequence stems from the rapidly increasing concentration of $\mathrm{CO}_{2}$ in the atmosphere since the mid 1800's. There is concern that, without proper carbon management, $\mathrm{CO}_{2}$ levels in the atmosphere may lead to unprecedented changes in the earth's climate, the “Greenhouse Effect.” Capture of $\mathrm{CO}_{2}$ from the atmosphere and storage in recalcitrant pools of plant biomass and soil (carbon sequestration) could provide a means of such carbon management. This feat may be possible through special management of forestry and agricultural practices as well as substitution of fossil fuels with biofuels. Such practices not only determine the rates of returning materials to the soil, but also the rates at which soil re-emits $\mathrm{CO}_{2}$ through decomposition processes. The cycle is very dynamic, and the balance is very sensitive to a variety of environmental conditions. A method of characterizing soil carbon systems in a timely fashion without disturbing the native character of the organic materials in the soil is therefore needed to evaluate the impact of such practices. The challenges of characterizing soil are vast because soil is a dynamic medium with regard to inanimate organic material and living organisms. Besides carbonates, the total organic material in soil is a mixture of plant and animal residues at varying stages of decomposition, of substances synthesized microbially or chemically from decomposition products, and of the remains of living bodies. ${ }^{1}$

There is presently a poor understanding of the roles of various compounds and organisms in terms of soil productivity and carbon cycling. The general classification of organic materials in soil includes low molecular weight organic acids, identifiable high molecular weight compounds, and humic substances. Low molecular weight organic acids can be synthesized by metabolic processes in plants (root exudates) or result from the decomposition of identifiable high molecular weight compounds (e.g. long-chain fatty acids, polysaccharides and proteins). They are rapidly degraded by microorganisms and, thus, have a transitory existence in soils. ${ }^{2}$ Organic acids routinely identified in soils include acetic, aconitic, benzoic, cinnamic, citric, formic, fumaric, gallic, lactic, maleic, malic, malonic, hydroxybenzoic, phthalic, protocatechuic, oxalic, salicyclic, succinic, tartaric and vanillic. ${ }^{3,4}$ Their abundance generally follows the sequence: aliphatic, aromatic and amino acids. ${ }^{5}$ Low molecular weight organic acids function as ligands, thus affecting soil properties and processes by chelating metals in solution and by ligand exchange at metal-hydroxide surfaces. ${ }^{6}$

Humic substances comprise a much more stable portion of organic materials in soils. It is an extraordinarily complex category, comprised of a heterogeneous mixtures of compounds ranging to > $300 \mathrm{kDa}$ molecular weight. No single chemical structure can be given to this category; in fact, there has never been a successful separation of humic

mixtures into discreet products. Rather, the components of humic mixtures are broadly categorized as humic acids (compounds soluble in water at $\mathrm{pH}>2$ ), fulvic acid (compounds soluble in water at any $\mathrm{pH}$ ) and humin (compounds insoluble in water at any $\mathrm{pH}$ ). True products of the soil, humic substances are defined as condensations of organic radicals produced through catabolism of plant and animal products within the soil. ${ }^{7}$ Their roles in the carbon cycle 
processes in soil are far reaching. In addition to providing buffer activity over a broad $\mathrm{pH}$ range, they influence several important physical properties, including aeration, aggregate stability, water capacity, and permeability. ${ }^{8}$ Clearly, this category deserves more definitive characterization.

Recently, characterization of various humic substances in soil samples has been accomplished through multistep analyses, including fractionization via ultrafiltration followed by elemental analysis, NMR $\left({ }^{1} \mathrm{H},{ }^{13} \mathrm{C},{ }^{31} \mathrm{P}\right)$, FT-IR, Raman scattering, and even SERS. ${ }^{9,10}$ However, this approach to soil analysis involved sample collection followed by laboratory-based refinement and analysis. Such steps can disturb the native character of a soil sample. Normal Raman analyses on refined humic samples have been performed using 514.5- and 647.1-nm laser excitation sources. ${ }^{11}$ Fourier transform Raman studies have also been pursued with 1064-nm excitation in efforts to reduce the incidence of interfering fluorescence signals from refined humic substances. ${ }^{12}$ SERS has the potential of minimizing sample refinement prior to analysis through not only a huge enhancement of Raman signal, but also selective adsorption of specific sample components, and even fluorescence quenching. Furthermore, the extremely narrow features of a Raman spectral signature can allow the characterization multiple sample components simultaneously. ${ }^{13}$

The SERS effect was first observed in1974 by Fleischmann and coworkers, who reported strongly enhanced Raman scatter from pyridine molecules adsorbed on electrochemically roughened silver electrodes. ${ }^{14}$ While they attributed the effect to increased surface area of the roughened electrode surface, later reports by the teams of Jeanmaire and Van Duyne, ${ }^{15}$ and Albrecht and Creighton ${ }^{16}$ not only confirmed the $10^{6}-10^{8}$-factor enhancement, but also attributed the enhancement to more complex surface enhancement processes. Since then, several models based on electromagnetic and chemical effects have been independently proposed. ${ }^{17-25}$ Based on these models, a variety of SERS substrate types and excitation sources have been applied towards the characterization of soil humic substances. SERS spectra have been reported for fulvic and humic acids adsorbed on copper electrodes , ${ }^{26}$ silver sols, ${ }^{10,27-29}$ and a silver nitratemodified Fe-C-Cr-Ni surface ${ }^{30}$ using visible laser excitation sources. Fourier transform SERS has been achieved with roughened gold electrodes and 1064-nm excitation. ${ }^{31,32}$ Other techniques in the application of Raman and SERS for soil humic substance analysis have been reviewed by Yang and Chase. ${ }^{33}$

In this work, we report the use of several solid surface-based SERS substrates in the detection of humic substances and low molecular weight compounds, including silver island films, redox-roughened copper foils, and silver- and goldcoated alumina substrates. The metal-coated alumina substrates represent a unique approach to SERS substrate development achieved in our laboratory- metal-coated dielectric microparticles. Briefly stated, a layer of dielectric micropartcles (0.5-5 $\mu \mathrm{m}$ diam.) is distributed on a solid support such as glass, quartz, filter paper or cellulosic membrane, and then coated with 50-150 nm of metal via thermal evaporation. Important surface parameters, including particle size, particle density, and silver thickness are easily controlled, thus yielding highly reproducible substrates which are both easy and economical to produce. Several dielectric particle materials, which impart the roughness 
critical to the SERS effect, have been investigated in our laboratory, including polystyrene, ${ }^{34}$ fumed silica, ${ }^{35}$ titanium dioxide ${ }^{36}$ and alumina. ${ }^{37}$ These substrates have been applied to the detection of a variety of environmentally significant compounds, including chlorinated pesticides, ${ }^{38}$ organophosphorus compounds, ${ }^{39}$ polycyclic aromatic compounds, ${ }^{40}$ and fungicides. ${ }^{41}$ The microparticle-based substrate technology has been incorporated in several fiberoptic probes used for remote detection. ${ }^{42,43}$ It has also been applied to genomics and biomedical analysis. ${ }^{44-46}$ In these studies, we have coupled alumina-based substrates with electrochemical processes for the detection of humic substances. In particular, we have observed that copper yields superior performance relative to silver as a metal coating for the alumina-based substrate when used at a holding potential of $-1.0 \mathrm{~V}$ (vs SCE). In a previous study using electrochemically roughened copper electrodes in an electrochemical cell, Liang et al. attributed a large SERS enhancement for humic substances to the specific binding of carboxylic ions and pyridine-substituted rings onto the copper surface. ${ }^{26}$ The copper-coated alumina substrate, reported herein for the first time, may prove to be an attractive alternative to redox-roughened electrodes due to the uniformity of the submicron surface features over relatively large areas, as well as the ease of reproducible fabrication.

\section{EXPERIMENTAL}

Instrumentation. The SERS spectra, unless otherwise stated, were collected from an electrochemical cell with the backscattering geometry illustrated in Figure 1. The 632.8-nm line a HeNe laser (Uniphase, Model 1145, Matecea, CA) was directed into the electrochemical cell with a small right angle prism (4mm X $4 \mathrm{~mm}$ face). The laser beam was focused onto the active surface of the SERS substrate, and the SERS signal was collected confocally with a 1”, f/1 lens. The power of the laser at the sample was approximately $8 \mathrm{~mW}$. Rayleigh scatter was rejected from the collected signal with a holographic notch filter (Kaiser) before transmission to the spectrograph. The spectrograph was a single-grating (600 gr/mm), 320-mm monochromator (ISA, Model HR-320) equipped with a red-enhanced intensified charge-coupled device (RE-ICCD). The spectral bandpass, defined by the 30-mm entrance slit, was $3.3 \mathrm{~cm}^{-1}$. The RE-ICCD (Princeton Instruments, Model RE/ICCD 576S) was thermoelectrically cooled to $-34^{\circ} \mathrm{C}$. Data acquisition was managed with CSMA v2.0 software, supplied by Princeton Instruments.

The electrochemical cell was a three-electrode system, assembled in a 2" diameter petri dish. The reference electrode for all electroSERS preparations and measurements was a saturated calomel electrode (SCE) (Analytical Sensors, Inc.). The counter electrode was a platinum wire, while a SERS substrate acted as the working electrode. The electrochemical cell parameters were controlled with a Bi-Potentiostat (Pine Instrument Company, Model AFRDE5, Grove City, PA),

SERS substrates. These studies involved silver-coated alumina-based substrates, silver-island substrates, copper foil substrates, and copper-coated alumina substrates. For both the silver- and copper-coated alumina-based substrates, the 
procedure for preparation was based on previous work on development of alumina-based substrates.(Bello et al., 1989b). Clean glass slides were spin-coated (2000 rpm; $20 \mathrm{~s})$ with $0.5 \%$ (wt/wt) alumina suspensions. The microparticle-coated slides were then coated with $1000 \AA$ of metal through e-beam evaporation in a high-vacuum system (Cook Vacuum Products; Model CVE 301E). Copper foil electrodes were made SERS-active through a RedOx roughing cycle performed in $0.1 \mathrm{M} \mathrm{KCl}$ solution. Prior to RedOx cycling, $1-\mathrm{cm}^{2}$ sections of the copper foil were polished to near-mirror finish with alumina lapping paper. In some cases where stated in the text, RedOx cycles were performed in the presence of analyte compounds, as well. Unless otherwise stated, a $-3.0 \mathrm{~V}-+3.0 \mathrm{~V}$ range was spanned by each of 25 RedOx cycles with a scan rate of $100 \mathrm{mV} / \mathrm{s}$.

Chemicals. Soil humic acid and fulvic acid standards were purchased from the International Humic Substance Society (IHSS). Prior to measurement, $10 \mathrm{~g} / \mathrm{L}(10,000 \mathrm{ppm})$ solutions were prepared by first dissolving the powders at $\mathrm{pH} 11$ (via addition of $0.1 \mathrm{M} \mathrm{NaOH}$ ). The stock solutions were then neutralized with $0.1 \mathrm{M} \mathrm{HCl}$. For electrochemical measurements, stock solutions were diluted to the reported concentrations in $0.1 \mathrm{M} \mathrm{KCl}$. Alcohol-based solutions of benzoic acid (Malickrodt), $p$-aminobenzoic acid (Aldrich), p-hydroxybenzoic acid (Matheson, Coleman and Bell), ophthalic acid (Phaltz and Bauer) and brilliant cresyl blue (Allied Chemical and Dye) were prepared with 200 proof ethanol (Aaper Alcohol and Chemical). All aqueous solutions or dilutions cited in the text were prepared with water that had been filtered with a Waters Milli-Q+ filtration system. The alumina used for the microparticle-based substrate preparations were graciously donated by Baikowski International. The nominal diameter of the special agglomerate-free alumina particles was $0.1 \mu \mathrm{m}$. Copper foil (Aldrich) used for the RedOx-roughened substrates was 0.065 ” thick.

\section{RESULTS AND DISCUSSION}

"Passive” SERS studies. The microparticle-based substrate technology developed in our laboratory has been used for the detection of a variety of chemicals of health and environmental interest. In nearly all cases the rough metal surface, usually silver, has provided a means for "passive” SERS measurements. In this approach, mere contact of the SERS substrates with analyte solutions has been adequate for detection of analytes at trace level concentrations. Figure 2 illustrates "passive" SERS spectra of compounds which would fit the low-molecular-weight category of organic components of soil, including benzoic acid, $p$-hydroxybenzoic acid, $p$-aminobenzoic acid, and $o$-phthalic acid. All spectra were acquired with alumina-based silver substrates. These SERS substrates were exposed to ethanol-based

solutions of the analytes, and spectra were recorded with 25-s acquisition times. Even though concentrations were in the mid-to-high ppm range, reasonable signal-to-noise is manifested by these spectra. Furthermore, the sharp spectral features of each spectrum clearly demonstrate the potential advantage of SERS for in-situ analysis of complex environments such as soil carbon systems. Because of the narrowness of the Raman bands, each spectrum has the capacity for a great deal of information, including the composition of complex samples and structures of sample 
components. Indeed, characterization of complex trace-level samples without pretreatment has been reported in other studies. ${ }^{13,47}$

While the detection of low-molecular-weight organic compounds via SERS is an encouraging result, the information yielded by observation of such end products can be quite limited in terms of characterizing soil carbon systems. Humic substances, on the other hand, not only comprise the most abundant class of organic compounds in soil, but also are the most dynamic in terms of chemical reactivity. Characterization of soil humic substances can therefore provide greater insight for determination of processes influencing soil carbon systems. Figure 3 illustrates the challenge of analyzing humic substances with Raman and SERS. An attempt at normal Raman measurement of Fulvic acid powder is shown in Figure 3a. Even though this spectrum was acquired with the relatively low-energy, 632.8-nm laser excitation source, it is dominated by a broadband emission from which little information can be extracted. In addition, "passive” SERS detection was applied to a 1000 ppm aqueous solution of humic acid, illustrated in Figure 3b. While some minor structures are discernable in the SERS spectrum, such features can be attributed to a combination of substrate background and the pixel signature of the ICCD.

\section{ElectroSERS studies}

RedOx-roughened copper foils. We have investigated the possibility of using electrochemically-enhanced SERS detection for analysis of humic substances. The motivation for this additional approach was the principle that specific holding potentials applied to a SERS substrate could promote the adsorption of certain molecules to the substrate surface, hence promoting enhanced, selective detection of humic components. Copper was selected as a substrate surface based on its potential reactivity with carboxylic acid groups, which are very abundant in humic substances such as humic acid. A common method of electrochemical SERS detection involves the use of RedOx-roughened metal foils. In this approach, the SERS substrate can be prepared in-situ through multiple reduction/oxidation cycles, which roughen the metal foil. Figure 4 illustrates SERS spectra of a 1000 ppm humic acid solution sample acquired with various holding potentials applied to a RedOx-roughened copper foil electrode. The electrode was roughened in-situ in the presence of $0.1 \mathrm{M} \mathrm{KCl}$. Prior to measurement, a $-3.0 \mathrm{~V}-+3.0 \mathrm{~V}$ range was cycled 25 times at a rate of $100 \mathrm{mV} / \mathrm{s}$. As demonstrated by the figure, the electrochemical enhancement is critical to humic acid detection. There is a clear dependence on holding potential for the observation of the broad D and G bands centered at approximately $1300 \mathrm{~cm}^{-1}$ and $1600 \mathrm{~cm}^{-1}$, respectively. The broadness of these bands is indicative of a complex, disordered carbon network. ${ }^{31}$ Virtually no structure was observed for holding potentials of $0.0 \mathrm{~V}$ and $-0.5 \mathrm{~V}$. For the discrete holding potentials tested, a dramatic increase in SERS signal was observed at more negative potentials, reaching a maximum at $-1.00 \mathrm{~V}$. (A less-than-optimum signal was observed at $-1.25 \mathrm{~V}$, not shown.)

In addition to holding potential, proper RedOx cycling parameters play a pivotal role in the electrochemical enhancement process. For example, Figure 5 demonstrates SERS spectra of the 1000 ppm humic acid sample, acquired 
at a $-1.0 \mathrm{~V}$ holding potential after $5,15,20$, and 25 cycles. Each cycle spanned a $-3.0 \mathrm{~V}-+3.0 \mathrm{~V}$ range with a rate of $100 \mathrm{mV} / \mathrm{s}$. A dramatic dependence on the number of cycles was observed, with maximum SERS activity yielded for 20 cycles. This optimized parameter corresponds to a substrate preparation time of approximately 40 min. This timeconsuming factor is a potential disadvantage of using RedOx-roughened foils, especially when preparing the foils insitu. Furthermore, in-situ preparation can influence the nature of the sample. While preparation of SERS-active foils can be performed in a separate environment from the sample, the ensuing handling or storage can be problematic due to the delicate nature of the specifically textured surface and vulnerability to air oxidation, especially for copper. Perhaps the greatest disadvantage of RedOx-roughened foils is the nonuniformity of SERS-active surface, resulting in variable signals observed across relatively small areas of the electrode. The limited reproducibility manifested by RedOxroughened metal foils can be attributed to the fact that preparation is based on chemical processes that may be influenced by environmental conditions as well as cleanliness and smoothness of the metal foil. These complications can severely compromise quantitative ability.

Hybrid electroSERS studies. We have investigated the use of the microparticle-based SERS substrate technology as an alternative to RedOx-roughened metal foils in a "hybrid electroSERS" technology. The SERS activity of microparticle-based substrate depends largely on the size of the microparticles, the density of the microparticle layer, and the thickness of the metal coating. These tangible parameters are readily controlled through the judicious selection of microparticles, the use of a programmable spin-coating system for depositing the microparticle layer on the substrate support, and the use of a vacuum evaporation system with a high-precision microbalance for the metal coating process. With no dependence on chemical processes, SERS substrate reproducibility is greatly enhanced relative to RedOxroughened foils. For example, the standard deviation for ten measurements acquired from various points of a relatively large $1 \mathrm{~cm}$ x $2.5 \mathrm{~cm}$ area of a microparticle-based substrate surface is routinely $<10 \%$. A successful integration of the microparticle-based substrate technology with the electroSERS technique described above could therefore greatly improve quantitative capability while exploiting the electrochemical enhancement. A critical factor in the integration would be an efficient coupling of the working electrode lead to the SERS-active surface. This feat was accomplished by positioning the lead wire at the bottom (glass) side of a copper-coated, alumina based substrate (see Figure 6). The wire was immobilized with a copper band, which was wrapped around a 1-cm long section of the substrate, allowing electrical contact between the wire and the rough copper surface of the opposite side of the substrate. The copper band was then wrapped in a heat shrink sleeve. As a result, the only metallic portion of the "hybrid electroSERS" electrode exposed to the sample solution was the SERS-active, copper-coated alumina surface.

SERS spectra of a $1000 \mathrm{ppm}$ soil humic acid solution acquired with the "hybrid electroSERS" electrode are illustrated in Figure 7. As with the previous studies with the RedOx-roughened copper foil, various holding potentials were tested. Consistent with the previous studies, a dramatic dependence of SERS signal on holding potential was observed. Maximum signal was observed for the $-1.0 \mathrm{~V}$ holding potential which yielded spectral features of structure 
and intensity comparable to those observed with the copper foil electrode. As a further demonstration of the dramatic SERS enhancement provided by the alumina-based copper substrate in the "hybrid electroSERS" electrode, Figure 8 depicts SERS spectra of a solution mixture of $1000 \mathrm{ppm}$ soil humic acid and $100 \mathrm{ppm}$ p-aminobenzoic acid (PABA). Spectra were acquired at various holding potentials. A weak spectrum for low-molecular-weight component, PABA, was observed even at $0.0 \mathrm{~V}$. As was the case for soil humic acid, the PABA signal was dramatically enhanced at the more negative potentials, indicating enhanced adsorption (likely via the carboxylic acid group). Nevertheless, features attributed to soil humic acid were discernable even in the presence of the dominant PABA spectrum acquired at a holding potential of $-1.0 \mathrm{~V}$. This result demonstrates the potential of the "hybrid electroSERS" technology for trace analysis of complex samples. Furthermore, the highly reproducible SERS-active surface of the copper-coated alumina substrate holds promise for improved quantitative capability with the electroSERS technique.

Electronic tuning for selective detection. A potential advantage unique to electroSERS would the ability to selectively detect or discriminate against certain components of complex samples by tuning the holding potential of the SERSactive electrode during measurements. This effect could arise from the selective adsorption or desorption of varying compounds based chemical functional group content. An example of this advantage is demonstrated in Figure 9, which shows spectra of an aqueous mixture of $1000 \mathrm{ppm}$ soil humic acid, $100 \mathrm{ppm}$ PABA and $1 \mathrm{ppm}$ brilliant cresyl blue at two different holding potentials. It is noteworthy that while both PABA and humic acid contain carboxylic functional groups, BCB does not. Regardless of the dramatic difference in the appearance of these spectra, both were acquired from identical samples with an identical RedOx-roughened copper foil electrode. At the $-0.5 \mathrm{~V}$ holding potential, the spectrum was completely dominated by the brilliant cresyl blue (BCB) component, even though the concentration of the BCB was at lest 100 times lower than either of the other mixture analytes. Unfortunately, this result demonstrates the possibility of severe background effects from relatively minor inteferents in complex samples. On the other hand, when a -1.25 V holding potential was applied to the SERS-active electrode, the BCB SERS signature was virtually eliminated from the mixture spectrum, allowing the observation of both PABA and soil humic acid spectral features. The fact that the PABA and humic acid bands are so weak yet still observable in the presence of such a spectacularly SERS-active component as BCB further emphasizes the discriminating ability of the electroSERS technique.

Raman and SERS studies have been initiated for determining the feasibility of their use in monitoring soil carbon systems. In these studies, we have selected some low molecular weight organic acids and some humic substances as model samples. Silver-coated alumina-based SERS substrates, which have a history of success for the detection of a variety of compounds of health and environmental interest, were effective in detecting the low molecular weight compounds through mere contact with the analyte solutions. However, this "passive” approach to SERS detection was unsuccessful in the analysis of soil humic acid samples. Consequently, an electroSERS approach has been evaluated for enhanced adsorption and detection of certain components of a very complex soil humic acid sample. Copper was selected as the SERS-active surface material based on its potential reactivity with carboxylic acid functional groups, which are so abundant in humic substances. Using the electroSERS approach in the analysis of aqueous samples, soil 
humic acid was successfully detected at 1000 ppm concentration when holding RedOx-roughened copper foil electrodes at $-1.0 \mathrm{~V}$. However, the general lack of signal reproducibility exhibited by the copper foil electrodes has motivated the development of a new "hybrid electroSERS" electrode, which integrates electrochemically enhanced adsorption with an alumina-based copper SERS substrate with highly uniform surface features. The "hybrid electroSERS" electrode yielded Raman enhancement comparable to that of the RedOx-roughened copper foil while offering dramatically improved signal reproducibility over relative large electrode surface areas. Hence, this new technology holds the promise of improved quantitative ability. Finally, the potential for discriminating against background signals from SERS-active interferents has been demonstrated through tuning the holding potential of electroSERS measurements.

\section{CONCLUSION}

Raman and SERS studies have been initiated for determining the feasibility of their use in monitoring soil carbon systems. In these studies, we have selected some low molecular weight organic acids and some humic substances as model samples. Silver-coated alumina-based SERS substrates, which have a history of success for the detection of a variety of compounds of health and environmental interest, were effective in detecting the low molecular weight compounds through mere contact with the analyte solutions. However, this "passive" approach to SERS detection was unsuccessful in the analysis of soil humic acid samples. Consequently, an electroSERS approach has been evaluated for enhanced adsorption and detection of certain components of a very complex soil humic acid sample. Copper was selected as the SERS-active surface material based on its potential reactivity with carboxylic acid functional groups, which are so abundant in humic substances. Using the electroSERS approach in the analysis of aqueous samples, soil humic acid was successfully detected at 1000 ppm concentration when holding RedOx-roughened copper foil electrodes at $-1.0 \mathrm{~V}$. However, the general lack of signal reproducibility exhibited by the copper foil electrodes has motivated the development of a new "hybrid electroSERS" electrode, which integrates electrochemically enhanced adsorption with an alumina-based copper SERS substrate with highly uniform surface features. The "hybrid electroSERS" electrode yielded Raman enhancement comparable to that of the RedOx-roughened copper foil while offering dramatically improved signal reproducibility over relative large electrode surface areas. Hence, this new technology holds the promise of improved quantitative ability. Finally, the potential for discriminating against background signals from SERS-active interferents has been demonstrated through tuning the holding potential of electroSERS measurements.

\section{ACKNOWLEDGEMENTS}

This research is jointly sponsored the Office of Biological and Environmental Research, U. S. Department of Energy under contract DE-AC05-00OR22725 with UT-Battelle, LLC. This work is also supported by the Laboratory Directed Research and Development Program at ORNL. In addition, D. L. Stokes is supported by an appointment to 
the Oak Ridge National Laboratory Postdoctoral Research Associates Program administered jointly by the Oak Ridge National Laboratory and Oak Ridge Institute for Science and Education. 


\section{REFERENCES}

1. Schnitzer, M. Soil Sci. 1991,151, 41-58.

2. Fox, T.R. In: Carbon Forms and Functions in Forest Soils, Kelly, J.M.; McFee, W.W., Eds., Soil Science Society of America: Madison, WI, 1995, pp 43-62.

3. Bruckert, S. Etudes de terrain Ann. Agron. 1970, 2, 421-452.

4. Kuiters, A.T.; Sarink, H.M. Soil Biol. Biochem. 1986, 18, 475-480.

5. Robert M.; Berthelin,J. In Interactions of Soil Minerals with Natural Organics and Microbes, Huang, P.M.; Schnitzer, M., Eds., Soil Science Society of America: Madison, WI, 1986, pp 453-495.

6. Stumm, W. Gioderma 1986, 38, 19-30.

7. Fisher, R.F. In: Carbon Forms and Functions in Forest Soils, Kelly, J.M.; McFee, W.W.,Eds., Soil Science Society of America: Madison, WI, 1995, pp 1-11.

8. Stevenson, F.J. Humus Chemistry: Genesis, Composition, Reactions, Wiley Interscience: New York, 1982.

9. Francioso, O.; Sanchez-Cortez, S.; Tugnoli, V.; Ciavatta, C.; Sitti, L.; Gessa, C. Appl. Spectrosc. 1996, 50, 11651174.

10. Francioso, O.; Sanchez-Cortez, S.; Tugnoli, V.; Ciavatta, C.; Gessa, C. Appl. Spectrosc. 1998, 52, 270-277.

11. Yang, Y.-H.; Li, B.-N.; Tao, Z.-Y. Spectrosc. Lett. 1994, 27, 649-660.

12. Yang, Y.-H.; Wang, T. Vibrat. Spectrosc. 1997, 14, 105-112.

13. Bello, J.M.; Stokes, D.L.; Vo-Dinh, T. Anal. Chem. 1990, 62, 1349-1352.

14. Fleischmann, M.J.; Hendra, P.J.; McQuillan, A.J. Chem. Phys. Lett. 1974, 26, 163-166.

15. Jeanmaire, D.J.; Van Duyne, R.P. J. Electroanal. Chem. 1977, 84, 1-20.

16. Albrecht, M.G.; Creighton, J.A. J. Am. Chem. Soc. 1977, 99, 5215-5217.

17. Chang, R.K.; Furtak T.E. (Eds) Surface-Enhanced Raman Scattering, Plenum Press: New York, 1982.

18. Kerker, M. Acc. Chem. Res. 1984, 17, 271-277.

19. Pockrand, I. Surface-Enhanced Raman Vibrational Studies at Solid/Gas Interfaces, Springer: Berlin, (1984).

20. Schatz, G.C. Acc. Chem. Res. 1984, 17, 370-376.

21. Wokaun, A. Solid State Physics 1984, 38, 223-294.

22. Moskovits, M. Rev. Mod. Phys. 1985, 57, 783-826.

23. Creighton, J.A. Surf. Sci. 1986, 173, 665-672.

24. Otto, A.; Mrozek, I.; Grabhorn H.; Akermann, W. J. Phys. Condens. Matter 1992, 4, 1143-1212.

25. Kambhampati, P.; Child, C.M.; Foster, M.C.; Campion, A. J. Chem. Phys. 1998, 108, 5013-5026.

26. Liang, E.J.; Yang, Y.; Kiefer, W. Spectrosc. Lett. 1999, 34, 689-701.

27. Yang, Y.-H.; Zhang, D.-H. Spectrosc. Lett. 1995, 28, 1203-1216.

28. Yang, Y.-H.; Zhou, Q.; Yu, J.-Y. J. Environ. Sci. Health 1996, A31, 1395-1405.

29. Sanchez- Cortez, S.; Francioso, O.; Ciavatta, C.; Garcia-Ramos, J.V.; Gessa, C. J. Coll. Interf. Sci. 1998, 198, 308318.

30. Liang, E.J.; Yang, Y.-H.; Kiefer, W. J. Environ. Sci. Health 1996, A31, 2477-2486.

31. Wang, T.; Zhong, F.-P.; Yang, Y.-H.; Zhang, D.-H. Spectrosc. Lett. 1996, 29, 1449-1458.

32. Wang, T.; Xiao, Y.-J.; Yang, Y.; Chase, H.A. J. Environ. Sci. Health 1999, A34, 749-765.

33. Yang, Y.; Chase, H.A. Spectrosc. Lett. 1998, 31, 821-848.

34. Moody, R.L.; Vo-Dinh, T.; Fletcher, W.H. Appl. Spectrosc. 1987, 41, 966-970.

35. Alak, A.M.; Vo-Dinh, T. Anal. Chem. 1989, 61, 656-660.

36. Bello, J.M.; Stokes, D.L.; Vo-Dinh, T. Anal Chem. 1989, 61, 1779-1783.

37. Bello, J.M.; Stokes, D.L.; Vo-Dinh, T. Appl. Spectrosc. 1989, 43, 1325-1330.

38. Alak, A.M.; Vo-Dinh, T. Anal. Chim. Acta 1988, 206, 333-337.

39. Alak, A.M.; Vo-Dinh, T. Anal. Chem. 1987, 59, 2149-2153.

40. Vo-Dinh, T.; Hiromoto, M.Y.K.; Begun, G.M.; Moody, R.L. Anal. Chem. 1984, 56, 1667-1670.

41. Narayanan, V.A.; Begun, G.M.; Stokes, D.L.; Sutherland, W.S.; Vo-Dinh, T. J. Raman Spectrosc. 1992, 23, 281286.

42. Bello, J.M.; Narayanan, V.A.; Stokes, D.L.; Vo-Dinh, T. Anal. Chem. 1990, 62, 2437-2441.

43. Stokes, D.L.; Vo-Dinh, T. Sensor Actuat. B-Chem. 2000, 69, 28-36.

44. Vo-Dinh, T.; Houke, K.; Stokes, D.L. Anal. Chem. 1994, 66, 3379-3383. 
45. Isola, N.R.; Stokes, D.L.; Vo-Dinh, T. Anal. Chem. 1998, 70, 1352-1356.

46. Vo-Dinh, T.; Stokes, D.L.; Griffin, G.D.; Volkan, M.; Kim, U.J.; Simon, M.I. J. Raman Spectrosc. 1999, 30, 785793.

47. Narayanan, V.A.; Bello, J.M.; Stokes, D.L.; Vo-Dinh, T. Analusis 1991, 19, 307-310. 


\section{FIGURE CAPTIONS:}

Figure 1. Schematic diagram of the SERS detection system.

Figure 2. SERS spectra of model compounds using alumina-based silver substrates.

Figure 3. Attempts at Raman scattering analysis of humic substances: a) normal Raman spectrum of fulvic acid powder, b) SERS spectrum of 1000 ppm humic acid acquired with alumina-based silver substrate.

Figure 4. SERS spectra of $1000 \mathrm{ppm}$ soil humic acid on RedOx-roughened copper foil electrodes at various holding potentials. Cycle parameters: $-3.0 \mathrm{~V}-3.0 \mathrm{~V}, 100 \mathrm{mV} / \mathrm{s}, 25$ cycles.

Figure 5. SERS spectra of $1000 \mathrm{ppm}$ soil humic acid acquired at $-1.0 \mathrm{~V}$ holding potential after various numbers of RedOx cycles with a copper foil electrode. Cycle parameters: $-3.0 \mathrm{~V}-3.0 \mathrm{~V}, 100 \mathrm{mV} / \mathrm{s}, 25$ cycles.

Figure 6. Schematic diagram of a hybrid electroSERS substrate based of copper-coated alumina.

Figure 7. SERS spectra of $1000 \mathrm{ppm}$ soil humic acid at various holding potentials using a hybrid electroSERS substrate, based on copper-coated alumina.

Figure 8. SERS spectra of a mixture of $1000 \mathrm{ppm}$ soil humic acid and $100 \mathrm{ppm}$ PABA at various holding potentials using a hybrid electroSERS substrate, based on copper-coated alumina.

Figure 9. SERS spectra of a mixture of $1000 \mathrm{ppm}$ soil humic acid, $100 \mathrm{ppm}$ PABA and $1 \mathrm{ppm}$ BCB at a) $-0.5 \mathrm{~V}$ holding potential, and b) $-1.25 \mathrm{~V}$ holding potential. Spectra were acquired with a RedOx-roughened copper foil electrode. Cycle parameters: $-3.0 \mathrm{~V}-3.0 \mathrm{~V}, 100 \mathrm{mV} / \mathrm{s}, 25$ cycles. 